\title{
Global Finite-Energy Solutions of the Maxwell-Schrödinger System
}

\author{
Yan Guo, Kuniaki Nakamitsu, Walter Strauss \\ ${ }^{1}$ Courant Institute, 251 Mercer Street, New York, NY 10012, USA \\ 2 Department of Natural Sciences, Tokyo Denki University, Saitama 350-03, Japan \\ 3 Department of Mathematics, Brown University, Providence, RI 02912, USA
}

Received: 11 April 1994

\begin{abstract}
The existence of global finite-energy solutions is proved for the initial value problem for the Maxwell-Schrödinger system in the Coulomb, Lorentz and temporal gauges
\end{abstract}

\section{Introduction}

We consider the coupled Maxwell-Schrödinger system in three space dimensions for a nonrelativistic charged particle in an electromagnetic field [4]. This system occurs as a model in laser physics [1]. Although, of course, the system is not Lorentz invariant, it is rotationally invariant and gauge invariant. In this paper we prove the existence of global finite-energy solutions of the initial value problem for the Maxwell-Schrödinger system in the Coulomb gauge.

K. Nakamitsu and M. Tsutsumi in [3 and 5] proved that the initial value problem for the Maxwell-Schrödinger system in the Lorentz gauge is globally well-posed in a space of smooth functions in dimensions one and two, and locally well-posed in dimension three. Y. Tsutsumi in [6] proved, by constructing the modified wave operator, that there exist global smooth solutions in the Coulomb gauge for a certain class of scattered data as $t \rightarrow+\infty$. However, the problem in three dimensions with initial condition at a finite time has remained open. The present paper resolves this existence question, but we have not succeeded in proving uniqueness.

In Sect. 2 we write the equations and introduce the approximate system to be used to make the construction. Essentially it is to replace the imaginary $i$ in the Schrödinger equation by $i+\varepsilon$ with a small dissipation constant $\varepsilon$. In Sect. 3 we construct global solutions of the approximate system in the Coulomb gauge. In Sect. 4 we pass to the limit $\varepsilon \rightarrow 0$, thereby obtaining global weak solutions. In Sect. 5 we prove the analogous result in the Lorentz gauge and in the temporal gauge.

This research was done primarily while all three authors were at Brown University. It was supported in part by NSF grant 90-23864 and ARO grant DAAH 04-93-G-0198. 
We will denote by $\|\cdot\|_{p}$ the norm in $L^{p}=L^{p}\left(\mathbb{R}^{3}\right)$, and by $\|\cdot\|_{k, p}$ the norm in the Sobolev space $W^{k, p}=W^{k, p}\left(\mathbb{R}^{3}\right)$. We write $H^{k}$ for $W^{k, 2}$ as usual, and $\mathbb{R}^{+}$ for the interval $[0, \infty)$. We denote the space of all weakly continuous mapping of $\mathbb{R}^{+}$to a Banach space $X$ by $C_{w}\left(\mathbb{R}^{+} ; X\right)$, and the space of all bounded weakly continuous mappings of $\mathbb{R}^{+}$to $X$ by $B C_{w}\left(\mathbb{R}^{+} ; X\right)$.

\section{The Maxwell-Schrödinger System}

We will use Greek indices $\mu, v, \ldots$ to run integers from 0 to 3 , Latin indices $j, k, \ldots$ to run integers from 1 to 3 , and the summation convention for repeated indices. We write $\partial_{0}$ for $\partial / \partial t, \partial_{j}$ for $\partial / \partial x^{j}$, and define $\partial^{\mu}$ by $\partial^{0}=\partial_{0}$ and $\partial^{j}=-\partial_{j}$.

A classical Maxwell-Schrödinger (MS) field in $3+1$ dimensional space-time consists of a vector potential with four real components $A_{\mu}$ and a complex scalar field $\psi$. After a suitable rescaling, we may write the equations of motion as

$$
\begin{aligned}
& \partial^{\mu} F_{\mu \nu}+J_{v}=0, \quad F_{\mu \nu}=\partial_{\mu} A_{v}-\partial_{v} A_{\mu}, \\
& i D_{0} \psi+D_{j} D_{j} \psi=0, \quad D_{\mu}=\partial_{\mu}-i A_{\mu},
\end{aligned}
$$

together with a gauge condition on the potential. Here the $J_{v}$ are the components of the charge-current density, given by

$$
J_{0}=|\psi|^{2}, \quad J_{j}=i\left(\bar{\psi} D_{j} \psi-\psi \overline{D_{j} \psi}\right) .
$$

We introduce a viscosity parameter $\varepsilon>0$, and regard the MS system (2.1)-(2.2) as the limit $\varepsilon \rightarrow 0$ of the regularized system

$$
\begin{gathered}
\partial^{\mu} F_{\mu v}+J_{v}+\varepsilon R_{v}=0, \\
D_{0} \psi-(i+\varepsilon) D_{j} D_{j} \psi=0,
\end{gathered}
$$

where

$$
R_{0}=0, \quad R_{j}=\partial_{j}\left\{|\psi|^{2}-2 \Delta^{-1}\left(\overline{D_{k} \psi} D_{k} \psi\right)\right\},
$$

with $\Delta^{-1} f=-(4 \pi r)^{-1} * f$. The terms $R_{v}$ are defined so that Eqs. (2.3) and (2.4) are compatible. In fact, $\partial^{\mu} \partial^{v} F_{\mu v}=0$ since $F_{\mu v}=-F_{v \mu}$. Thus (2.3) requires the equation of continuity

$$
\partial^{v}\left(J_{v}+\varepsilon R_{v}\right)=0
$$

But by (2.4),

$$
\begin{aligned}
\partial^{v} J_{v} & =\partial_{0}|\psi|^{2}-2 \operatorname{Re}\left\{\mathrm{i} \partial_{j}\left(\bar{\psi} D_{j} \psi\right)\right\} \\
& =2 \operatorname{Re}\left\{\bar{\psi}\left(D_{0} \psi-\mathrm{i} D_{j} D_{j} \psi\right)\right\}=2 \varepsilon \operatorname{Re}\left\{\bar{\psi} D_{j} D_{j} \psi\right\} .
\end{aligned}
$$

Thus (2.6) implies $\partial^{v} R_{v}=-2 \operatorname{Re}\left\{\bar{\psi} D_{j} D_{j} \psi\right\}$. This is the compatibility condition for $R_{v}$. It has the solution (2.5).

We define the charge $Q$ and the energy $E$ by

$$
\begin{aligned}
& Q=\int|\psi|^{2} d x, \\
& E=\int\left\{\overline{D_{j} \psi} D_{j} \psi+\frac{1}{4} F_{\mu \nu} F_{\mu \nu}\right\} d x .
\end{aligned}
$$


Lemma 2.1. Let $\psi$ and $A_{\mu}$ be smooth functions on $[0, T] \times \mathbb{R}^{3}$ satisfying (2.3) and (2.4). Then we have

$$
\begin{gathered}
Q(T)+2 \varepsilon \int_{0}^{T} d t \int \widehat{D_{j} \psi} D_{j} \psi d x=Q(0), \\
E(T)+2 \varepsilon \int_{0}^{T} d t \int\left\{\overline{D_{j} D_{j} \psi} D_{k} D_{k} \psi d x+V|\psi|^{2}\right\} d x=E(0),
\end{gathered}
$$

where $V$ is the nonnegative function defined by

$$
V=\frac{1}{2}|\psi|^{2}-\Delta^{-1}\left(\overline{D_{j} \psi} D_{j} \psi\right) .
$$

Proof. From (2.4) we have

$$
\frac{d Q}{d t}=2 \operatorname{Re} \int \bar{\psi} D_{0} \psi d x=2 \varepsilon \operatorname{Re} \int \bar{\psi} D_{j} D_{j} \psi d x=-2 \varepsilon \int \overline{D_{j} \psi} D_{j} \psi d x
$$

This implies (2.7). Similarly, from (2.4),

$$
\begin{aligned}
\frac{d}{d t} \int \overline{D_{j} \psi} D_{j} \psi d x & =2 \operatorname{Re} \int \overline{D_{j} \psi} D_{0} D_{j} \psi d x \\
& =2 \operatorname{Re} \int \overline{D_{j} \psi}\left(D_{j} D_{0}+i F_{j 0}\right) \psi d x \\
& =2 \operatorname{Re} \int\left\{-\overline{D_{j} D_{j} \psi} D_{0} \psi+i F_{j 0} \overline{D_{j} \psi} \psi\right\} d x \\
& =-\int\left\{2 \varepsilon \overline{D_{j} D_{j} \psi} D_{k} D_{k} \psi d x+F_{j 0} J_{j}\right\} d x
\end{aligned}
$$

On the other hand, using the identity $\partial_{0} F_{j k}+\partial_{j} F_{k 0}+\partial_{k} F_{0 j}=0$ and (2.3), we have

$$
\begin{aligned}
\frac{d}{d t} \int \frac{1}{4} F_{\mu \nu} F_{\mu \nu} d x & =\int\left\{F_{j 0} \partial_{0} F_{j 0}+\frac{1}{2} F_{j k} \partial_{0} F_{j k}\right\} d x \\
& =\int\left\{F_{j 0} \partial_{0} F_{j 0}-\frac{1}{2} F_{j k}\left(\partial_{j} F_{k 0}+\partial_{k} F_{0 j}\right)\right\} d x \\
& =-\int F_{j 0} \partial^{\mu} F_{\mu j} d x=\int F_{j 0}\left(J_{j}+\varepsilon R_{j}\right) d x \\
& =\int\left\{F_{j 0} J_{j}-2 \varepsilon \hat{\partial}_{j} F_{j 0} V\right\} d x \\
& =\int\left\{F_{j 0} J_{j}+2 \varepsilon \partial^{\mu} F_{\mu 0} V\right\} d x \\
& =\int\left\{F_{j 0} J_{j}-2 \varepsilon V|\psi|^{2}\right\} d x
\end{aligned}
$$

Adding the above two results, we deduce (2.8).

\section{Regularized MS Field in the Coulomb Gauge}

We begin by constructing global solutions of the initial value problem for the regularized system (2.3)-(2.4) in the Coulomb gauge $\partial_{j} A_{j}=0$. In this gauge, Eq. (2.3) for $v=0$ and $v=j \neq 0$ reduce to the equations

$$
\begin{gathered}
\Delta A_{0}=J_{0}, \\
\square A_{j}=\partial_{j} \partial_{0} A_{0}-J_{j}-\varepsilon R_{j},
\end{gathered}
$$


respectively. These equations may be written as follows. By (3.1), we may set $A_{0}=\Delta^{-1} J_{0}$ in (2.4). Furthermore, using (2.6) and (2.5), we have

$$
\begin{gathered}
\partial_{j} \partial_{0} A_{0}=\partial_{j} \partial_{0} \Delta^{-1} J_{0}=\partial_{j} \partial_{k} \Delta^{-1}\left(J_{k}+\varepsilon R_{k}\right) \\
=\partial_{j} \partial_{k} \Delta^{-1} J_{k}+\varepsilon R_{j} .
\end{gathered}
$$

Thus the system (2.3)-(2.4) in the Coulomb gauge reduces to the following system for the fields $A=\left(A_{1}, A_{2}, A_{3}\right)$ and $\psi$ :

$$
\begin{gathered}
\square A=-P J, \quad \nabla \cdot A=0, \\
\partial_{0} \psi-(i+\varepsilon) \Delta \psi=K_{\varepsilon},
\end{gathered}
$$

where $J=\left(J_{1}, J_{2}, J_{3}\right), P J=J-\nabla\left(\nabla \cdot \Delta^{-1} J\right)$ is the projection of $J$ on the divergence-free vector fields, and

$$
K_{\varepsilon}=i\left(\Delta^{-1} J_{0}\right) \psi-(i+\varepsilon)(2 i A \cdot \nabla \psi+A \cdot A \psi) .
$$

We consider the system (3.3)-(3.4) with the initial condition

$$
\left.\left(A, \partial_{0} A, \psi\right)\right|_{t=0}=(a, b, \phi) .
$$

The Coulomb gauge condition imposes the initial constraints

$$
\nabla \cdot a=\nabla \cdot b=0
$$

The main result of this section is the following.

Lemma 3.1. Let $\varepsilon>0$ and $k \geqq 1$. Assume that $(a, b, \phi) \in H^{k} \times H^{k-1} \times H^{k}$ and $a, b$ satisfy (3.6). Then there is a unique solution $(A, \psi)$ of (3.3)-(3.5) such that

$$
\left(A, \partial_{0} A, \psi\right) \in C\left(\mathbb{R}^{+} ; H^{k} \times H^{k-1} \times H^{k}\right), \quad \nabla \psi \in L^{2}\left(\mathbb{R}^{+} ; H^{k}\right) .
$$

Moreover, we have

$$
\|A(t)\|_{2}<C\{1+t\}, \quad \sum_{\mu=0}^{3}\left\|\partial_{\mu} A(t)\right\|_{2}<C, \quad\|\psi(t)\|_{1,2}<C,
$$

for some constants $C=C\left(\|a\|_{1,2},\|b\|_{2},\|\phi\|_{1,2}\right)$ independent of $t$ and $\varepsilon$.

To prove the lemma, we write the wave equation in (3.3) as the pair of equations

$$
\partial_{0} A=B, \quad \partial_{0} B-\Delta A=-P J .
$$

We convert the system consisting of this pair, of (3.4), and of the initial condition (3.5) to the integral equation

$$
u=G_{\Phi} N(u) .
$$

Here $\Phi=(a, b, \phi), u=(A, B, \psi), N(u)=\left(0,-P J, K_{\varepsilon}\right)$, and $G_{\Phi}$ is the linear mapping $(A, B, \psi) \rightarrow\left(A^{G}, B^{G}, \psi^{G}\right)$ defined as

$$
\begin{gathered}
\left(\begin{array}{c}
A^{G}(t) \\
B^{G}(t)
\end{array}\right)=M(t)\left(\begin{array}{l}
a \\
b
\end{array}\right)+\int_{0}^{t} M(t-s)\left(\begin{array}{l}
A(s) \\
B(s)
\end{array}\right) d s, \\
\psi^{G}(t)=S(t) \phi+\int_{0}^{t} S(t-s) \psi(s) d s,
\end{gathered}
$$


where

$$
M(t)=\exp \left\{t\left(\begin{array}{cc}
0 & I \\
\Delta & 0
\end{array}\right)\right\}, \quad S(t)=\exp \{(i+\varepsilon) t \Delta\}
$$

The operator $P$ is the projection of the space of real 3 -vectors in $H^{k}$ onto its subspace consisting of divergence-free vectors. We denote this subspace as $H_{\sigma}^{k}$. We define $X_{0}^{k}$ to be the space of all triples $\Phi=(a, b, \phi)$ in $H_{\sigma}^{k} \times H_{\sigma}^{k-1} \times H^{k}$, with the norm

$$
\|\Phi\|_{X_{0}^{k}}=\|a\|_{k, 2}+\|b\|_{k-1,2}+\|\phi\|_{k, 2} .
$$

Let $T>0$, let $I=[0, T]$, and let $\|\cdot\|_{k, p, q}$ be the norm in $L^{q}\left(I ; W^{k, p}\right)$. As a solution space for the integral equation (3.8), we take the space $X^{k}(T)$ of all triples $u=(A, B, \psi)$ in $C\left(I ; X_{0}^{k}\right)$ such that $\nabla \psi$ is in $L^{2}\left(I ; H^{k}\right)$, with the norm defined by

$$
\|u\|_{X^{k}(T)}=\|A\|_{k, 2, \infty}+\|B\|_{k-1,2, \infty}+\|\psi\|_{k, 2, \infty}+\|\nabla \psi\|_{k, 2,2} .
$$

We also introduce the space $Y^{k}(T)$ of all $u=(A, B, \psi)$ in $L^{2}\left(I ; H_{\sigma}^{k} \times H_{\sigma}^{k-1} \times\right.$ $W^{k, 3 / 2}$ ), with the norm

$$
\|u\|_{Y^{k}(T)}=\|A\|_{k, 2,2}+\|B\|_{k-1,2,2}+\|\psi\|_{k, 3 / 2,2} .
$$

We write $X^{k}(T), Y^{k}(T)$ simply as $X^{k}, Y^{k}$ when there is no danger of confusion.

Lemma 3.2. Let $\varepsilon>0$. Let $k \geqq 1$ and $\Phi \in X_{0}^{k}$. Then $G_{\Phi}$ maps $Y^{k}$ to $X^{k}$, with

$$
\left\|G_{\Phi} u\right\|_{X^{k}} \leqq C_{\varepsilon}\|\Phi\|_{X_{0}^{k}}+C_{\varepsilon}\left\{T^{1 / 4}+T^{1 / 2}\right\}\|u\|_{Y^{k}}
$$

Proof. Let $u=(A, B, \psi) \in Y^{k}$, and let $\left(A^{G}, B^{G}, \psi^{G}\right)$ be defined by (3.9)-(3.10). Note that $M(t)$ is an isometry on $H_{\sigma}^{k} \times H_{\sigma}^{k-1}$. Therefore, from (3.9) we have $\left(A^{G}, B^{G}\right) \in C\left(I ; H_{\sigma}^{k} \times H_{\sigma}^{k-1}\right)$, with

$\left\|A^{G}\right\|_{k, 2, \infty}+\left\|B^{G}\right\|_{k-1,2, \infty} \leqq C\left\{\|a\|_{k, 2}+\|b\|_{k-1,2}\right\}+C T^{1 / 2}\left\{\|A\|_{k, 2,2}+\|B\|_{k-1,2,2}\right\}$.

Thus to complete the proof, it is sufficient to show that $\psi^{G}$ is in $C\left(I ; H^{k}\right)$ and satisfies

$$
\left\|\psi^{G}\right\|_{k, 2, \infty}+\left\|\nabla \psi^{G}\right\|_{k, 2,2} \leqq C_{\varepsilon}\left\{\|\phi\|_{k, 2}+T^{1 / 4}\|\psi\|_{k, 3 / 2,2}\right\}
$$

To see this, we may assume $k=0$. Now $\psi_{0}=S(t) \phi$ is the solution of the initial value problem

$$
\partial_{0} \psi_{0}-(i+\varepsilon) \Delta \psi_{0}=0,\left.\quad \psi_{0}\right|_{t=0}=\phi .
$$

The standard energy-type estimate shows

$$
\|S(t) \phi\|_{2}^{2}+2 \varepsilon \int_{0}^{t}\|\nabla S(t) \phi\|_{2}^{2} d s=\|\phi\|_{2}^{2} .
$$

On the other hand, the standard $L^{p}-L^{q}$ estimate for the heat evolution operator $e^{\varepsilon t \Delta}$ implies

$$
\begin{gathered}
\|S(t) f\|_{2} \leqq C_{\varepsilon} t^{-1 / 4}\|f\|_{3 / 2}, \\
\|\nabla S(t) f\|_{2} \leqq C_{\varepsilon} t^{-3 / 4}\|f\|_{3 / 2} .
\end{gathered}
$$


By (3.13), we have

$$
\begin{aligned}
\left\|\int_{0}^{t} S(t-s) \psi(s) d s\right\|_{2} & \leqq C_{\varepsilon} \int_{0}^{t}(t-s)^{-1 / 4}\|\psi(s)\|_{3 / 2} d s \\
& \leqq C_{\varepsilon} t^{1 / 4}\left\{\int_{0}^{t}\|\psi(s)\|_{3 / 2}^{2} d s\right\}^{1 / 2}
\end{aligned}
$$

and it follows that $\psi^{G} \in C\left(I ; L^{2}\right)$. By (3.14) and the singular integral inequality, we also have

$$
\begin{aligned}
\int_{0}^{t}\left\|\nabla \int_{0}^{\tau} S(\tau-s) \psi(s) d s\right\|_{2}^{2} d \tau & \leqq C_{\varepsilon} \int_{0}^{t}\left\{\int_{0}^{\tau}(\tau-s)^{-3 / 4}\|\psi(s)\|_{3 / 2} d s\right\}^{2} d \tau \\
& \leqq C_{\varepsilon}\left\{\int_{0}^{t}\|\psi(s)\|_{3 / 2}^{4 / 3} d s\right\}^{3 / 2} \\
& \leqq C_{\varepsilon} t^{1 / 2} \int_{0}^{t}\|\psi(s)\|_{3 / 2}^{2} d s
\end{aligned}
$$

From (3.12), (3.15) and (3.16), we deduce (3.11) for $k=0$.

We next show that the nonlinear term $N(u)$ is locally Lipschitz as a mapping from $X^{k}$ to $Y^{k}$ for $k \geqq 1$. To this end, we recall the Gagliardo-Nirenberg inequality

$$
\left\|d^{j} f\right\|_{p} \leqq C\left\|d^{k} f\right\|_{q}^{s}\|f\|_{r}^{1-s}
$$

where $1 / p-j / 3=s(1 / q-k / 3)+(1-s) / r, j / k \leqq s \leqq 1$ (if $k-j-3 / q$ is a nonnegative integer, only $s<1$ is allowed), and where

$$
\left\|d^{j} f\right\|_{p}=\left\{\sum_{|\alpha|=j}\left\|\frac{\partial^{\alpha f}}{\partial x^{\alpha}}\right\|_{p}\right\}^{1 / p} .
$$

We will also use the estimate

$$
\left\|\Delta^{-1} f\right\|_{6} \leqq C\|f\|_{6 / 5}
$$

which follows for instance from applying the generalized Young's inequality to the expression $\Delta^{-1} f=(4 \pi r)^{-1} * f$.

Lemma 3.3. Let $k \geqq 1$. Then $N(u)$ maps $X^{k}$ to $Y^{k}$. We have

$$
\begin{gathered}
\|N(u)\|_{Y^{k}} \leqq\left(1+T^{1 / 2}\right) Z_{\varepsilon, k}\left(\|u\|_{X^{1}}\right)\|u\|_{X^{k}} \\
\left\|N(u)-N\left(u^{\prime}\right)\right\|_{Y^{k}} \leqq\left(1+T^{1 / 2}\right) Z_{\varepsilon, k}\left(\|u\|_{X^{k}}+\left\|u^{\prime}\right\|_{X^{k}}\right)\left\|u-u^{\prime}\right\|_{X^{k}}
\end{gathered}
$$

where $Z_{\varepsilon, k}(r)=C_{\varepsilon, k}\left\{r+r^{2}\right\}$.

Proof. Let $u=(A, B, \psi) \in X^{k}$. We recall the definition $N(u)=\left(0,-P J, K_{\varepsilon}\right)$. We will estimate the components of $N(u)$ using (3.17) and (3.18). We recall that $J_{j}=2 \operatorname{Re}\left\{i \bar{\psi} D_{j} \psi\right\}$. We first show that

$$
\|J\|_{k-1,2,2} \leqq C\left\{1+T^{1 / 2}\right\}\left\{\|u\|_{X^{1}}+\|u\|_{X^{1}}^{2}\right\}\|u\|_{X^{k}} .
$$


Indeed, let $f_{1}=\bar{\psi} \nabla \psi$ and $f_{2}=A\|\psi\|^{2}$. The $L^{2}$-norms of $f_{1}$ and $f_{2}$ are estimated as

$$
\begin{aligned}
& \left\|f_{1}\right\|_{2} \leqq\|\psi\|_{6}\|\nabla \psi\|_{3} \leqq C\|\psi\|_{1,2}\|\nabla \psi\|_{1,2} \\
& \left\|f_{2}\right\|_{2} \leqq\|A\|_{6}\|\psi\|_{6}^{2} \leqq C\|A\|_{1,2}\|\psi\|_{1,2}^{2} .
\end{aligned}
$$

These give

$$
\begin{aligned}
\|J\|_{0,2,2} & \leqq\|\psi\|_{1,2, \infty}\|\nabla \psi\|_{1,2,2}+T^{1 / 2}\|A\|_{1,2, \infty}\|\psi\|_{1,2, \infty}^{2} \\
& \leqq C\left\{1+T^{1 / 2}\right\}\left\{\|u\|_{X^{1}}^{2}+\|u\|_{X^{1}}^{3}\right\} .
\end{aligned}
$$

We also have the estimates

$$
\begin{aligned}
\left\|d^{k} f_{1}\right\|_{2} & \leqq C \sum_{a+b=k}\left\|d^{a} \psi\right\|_{4}\left\|d^{b} \nabla \psi\right\|_{4} \\
& \leqq C \sum_{a+b=k}\left\|d^{k} \psi\right\|_{4}^{a / k}\|\psi\|_{4}^{1-a / k}\left\|d^{k} \nabla \psi\right\|_{4}^{b / k}\|\nabla \psi\|_{4}^{1-b / k} \\
& \leqq C \sum_{a+b=k}\|\psi\|_{k+1,2}^{a / k}\|\psi\|_{1,2}^{1-a / k}\|\nabla \psi\|_{k+1,2}^{b / k}\|\nabla \psi\|_{1,2}^{1-b / k},
\end{aligned}
$$

and

$$
\begin{aligned}
\left\|d^{k} f_{2}\right\|_{2} & \leqq C \sum_{a+b+c=k}\left\|d^{a} A\right\|_{6}\left\|d^{b} \psi\right\|_{6}\left\|d^{c} \psi\right\|_{6} \\
& \leqq C \sum_{a+b=k}\left\|d^{k} A\right\|_{6}^{a / k}\|A\|_{6}^{1-a / k}\left\|d^{k} \psi\right\|_{6}^{b / k}\|\psi\|_{6}^{2-b / k} \\
& \leqq C \sum_{a+b=k}\|A\|_{k+1,2}^{a / k}\|A\|_{1,2}^{1-a / k}\|\psi\|_{k+1,2}^{b / k}\|\psi\|_{1,2}^{2-b / k} \\
& \leqq C\left\{\|A\|_{1,2}+\|\psi\|_{1,2}\right\}^{2}\left\{\|A\|_{k+1,2}+\|\psi\|_{k+1,2}\right\}
\end{aligned}
$$

These imply

$$
\begin{aligned}
\left\|d^{k} f_{1}\right\|_{0,2,2} & \leqq C \sum_{a+b=k}\|\psi\|_{k+1,2, \infty}^{a / k}\|\psi\|_{1,2, \infty}^{1-a / k}\|\nabla \psi\|_{k+1,2,2}^{b / k}\|\nabla \psi\|_{1,2,2}^{1-b / k} \\
& \leqq C\left\{\|\psi\|_{1,2, \infty}+\|\nabla \psi\|_{1,2,2}\right\}\left\{\|\psi\|_{k+1,2, \infty}+\|\nabla \psi\|_{k+1,2,2}\right\}
\end{aligned}
$$

and

$$
\left\|d^{k} f_{2}\right\|_{0,2,2} \leqq C T^{1 / 2}\left\{\|A\|_{1,2, \infty}+\|\psi\|_{1,2, \infty}\right\}^{2}\left\{\|A\|_{k+1,2, \infty}+\|\psi\|_{k+1,2, \infty}\right\},
$$

respectively. Thus

$$
\left\|d^{k} J\right\|_{0,2,2} \leqq C\left\{1+T^{1 / 2}\right\}\left\{\|u\|_{X^{1}}+\|u\|_{X^{1}}^{2}\right\}\|u\|_{X^{k+1}} .
$$

This estimate, together with (3.22), proves (3.21) for all $k \geqq 1$.

We will next show that

$$
\left\|K_{\varepsilon}\right\|_{k, 3 / 2,2} \leqq C\left\{1+T^{1 / 2}\right\}\left\{\|u\|_{X^{1}}+\|u\|_{X^{1}}^{2}\right\}\|u\|_{X^{k}} .
$$

We recall the definition $K_{\varepsilon}=i g_{1}-(i+\varepsilon)\left(2 i g_{2}+g_{3}\right)$, where $g_{1}=\left(\Delta^{-1} J_{0}\right) \psi$, $g_{2}=A \cdot \nabla \psi$, and $g_{3}=A \cdot A \psi$. Using (3.18), we have

$$
\begin{aligned}
\left\|g_{1}\right\|_{3 / 2} & \leqq\left\|\Delta^{-1} J_{0}\right\|_{6}\|\psi\|_{2} \leqq C\left\|J_{0}\right\|_{6 / 5}\|\psi\|_{2} \\
& \leqq C\|\psi\|_{3}\|\psi\|_{2}^{2} \leqq C\|\psi\|_{1,2}^{3},
\end{aligned}
$$


$\left\|g_{2}\right\|_{3 / 2} \leqq\|A\|_{6}\|\nabla \psi\|_{2} \leqq C\|\nabla A\|_{2}\|\nabla \psi\|_{2}$,

$\left\|g_{3}\right\|_{3 / 2} \leqq\|A\|_{6}\|A\|_{6}\|\nabla \psi\|_{3} \leqq C\|\nabla A\|_{2}^{2}\|\psi\|_{1,2}$.

These estimates yield

$$
\begin{aligned}
\left\|K_{\varepsilon}\right\|_{0,3 / 2,2} & \leqq C T^{1 / 2}\left\{\|\psi\|_{1,2, \infty}^{2}+\|A\|_{1,2, \infty}+\|A\|_{1,2, \infty}^{2}\right\}\|\psi\|_{1,2, \infty} \\
& \leqq C T^{1 / 2}\left\{\|u\|_{X^{1}}^{2}+\|u\|_{X^{1}}^{3}\right\} .
\end{aligned}
$$

We also have the following estimates for the derivatives,

$$
\begin{aligned}
\left\|d^{k} g_{1}\right\|_{3 / 2} & \leqq \sum_{a+b=k}\left\|d^{a} \Delta^{-1} J_{0}\right\|_{6}\left\|d^{b} \psi\right\|_{2} \\
& \leqq C\left\|J_{0}\right\|_{6 / 5}\left\|d^{k} \psi\right\|_{2}+\sum_{\substack{a+b=k \\
a \neq 0}}\left\|d^{a} J_{0}\right\|_{6 / 5}\left\|d^{b} \psi\right\|_{2} \\
& \leqq C\|\psi\|_{1,2}^{2}\|\psi\|_{k, 2}+C \sum_{\substack{a+b=k \\
a \neq 0}}\left\{\sum_{i+j=a}\left\|d^{1} \psi\right\|_{6 a /(2 a+1)}\left\|d^{j} \psi\right\|_{6 a /(2 a+j)}\right\}\left\|d^{b} \psi\right\|_{2} \\
& \leqq C\|\psi\|_{1,2}^{2}\|\psi\|_{k, 2}+C \sum_{\substack{a+b=k \\
a \neq 0}}\left\|d^{a} \psi\right\|_{2}\|\psi\|_{3}\left\|d^{b} \psi\right\|_{2} \\
& \leqq C\|\psi\|_{1,2}^{2}\|\psi\|_{k, 2}, \quad \\
\left\|d^{k} g_{2}\right\|_{3 / 2} & \leqq C \sum_{a+b=k}\left\|d^{a} A\right\|_{6 k /(k+2 a)}\left\|d^{b} \nabla \psi\right\|_{6 k /(k+2 b)}
\end{aligned}
$$$$
\leqq C \sum_{a+b=k}\left\|d^{k} A\right\|_{2}^{a / k}\|A\|_{6}^{1-a / k}\left\|d^{k} \nabla \psi\right\|_{2}^{b / k}\|\nabla \psi\|_{6}^{1-b / k}
$$$$
\leqq C \sum_{a+b=k}\|A\|_{k, 2}^{a / k}\|A\|_{1,2}^{1-a / k}\|\nabla \psi\|_{k, 2}^{b / k}\|\nabla \psi\|_{1,2}^{1-b / k},
$$

$\left\|d^{k} g_{3}\right\|_{3 / 2} \leqq C \sum_{a+b+c=k}\left\|d^{a} A\right\|_{6 k /(k+2 a)}\left\|d^{b} A\right\|_{6 k /(k+2 b)}\left\|d^{c} \psi\right\|_{3 k / c}$

$$
\begin{aligned}
& \leqq C \sum_{a+b=k}\left\|d^{k} A\right\|_{2}^{a / k}\|A\|_{6}^{2-a / k}\left\|d^{k} \psi\right\|_{3}^{b / k}\|\psi\|_{\infty}^{1-b / k} \\
& \leqq C \sum_{a+b=k}\|A\|_{k, 2}^{a / k}\|A\|_{1,2}^{2-a / k}\|\nabla \psi\|_{k, 2}^{b / k}\|\nabla \psi\|_{1,2}^{1-b / k} .
\end{aligned}
$$

When we take the $L^{2}$-norm in time, we obtain

$$
\begin{aligned}
\left\|d^{k} g_{1}\right\|_{0,3 / 2,2} & \leqq C T^{1 / 2}\|\psi\|_{k, 2, \infty}\|\psi\|_{0,2, \infty}^{2}, \\
\left\|d^{k} g_{2}\right\|_{0,3 / 2,2} & \leqq C \sum_{a+b=k}\|A\|_{k, 2, \infty}^{a / k}\|A\|_{1,2, \infty}^{1-a / k}\|\nabla \psi\|_{k, 2,2}^{b / k}\|\nabla \psi\|_{1,2,2}^{1-b / k} \\
& \leqq C\left\{\|A\|_{k, 2, \infty}+\|\nabla \psi\|_{k, 2,2}\right\}\left\{\|A\|_{1,2, \infty}+\|\nabla \psi\|_{1,2,2}\right\}, \\
\left\|d^{k} g_{3}\right\|_{0,3 / 2,2} & \leqq C \sum_{a+b=k}\|A\|_{k, 2, \infty}^{a / k}\|A\|_{1,2, \infty}^{2-a / k}\|\nabla \psi\|_{k, 2,2}^{b / k}\|\nabla \psi\|_{1,2,2}^{1-b / k} \\
& \leqq C\left\{\|A\|_{k, 2, \infty}+\|\nabla \psi\|_{k, 2,2}\right\}\left\{\|A\|_{1,2, \infty}+\|\nabla \psi\|_{1,2,2}\right\}^{2},
\end{aligned}
$$

respectively. Thus

$$
\left\|d^{k} K_{\varepsilon}\right\|_{0,3 / 2,2} \leqq C\{1+\varepsilon\}\left\{1+T^{1 / 2}\right\}\left\{\|u\|_{X^{1}}+\|u\|_{X^{1}}^{2}\right\}\|u\|_{X^{k}} .
$$


This estimate, together with (3.24), proves (3.23). From (3.21) and (3.23), we have (3.19). The proof of (3.20) is similar.

Lemma 3.4. Let $k \geqq 1$ and $\Phi \in X_{0}^{k}$. Then there is a $T_{\max }>0$ such that the equation $u=G_{\Phi} N(u)$ has a unique solution $u$ in $X^{k}(T)$ for all $T \in\left(0, T_{\max }\right)$, and such that if $T_{\max }<\infty$, then

$$
\varlimsup_{t \uparrow T}\|u(t)\|_{X_{0}^{1}}=\infty
$$

Moreover, if $\Phi_{n} \in X_{0}^{k}$ with $\Phi_{n} \rightarrow \Phi$ in $X_{0}^{k}$, and if $T \in\left(0, T_{\max }\right)$, then the solution $u_{n}$ of (3.8) with $\Phi$ replaced by $\Phi_{n}$ belongs to $X^{k}(T)$ for sufficiently large $n$, and $u_{n} \rightarrow u$ in $X^{k}(T)$.

Proof. By Lemmas 3.2 and 3.3, we have

$$
\begin{gathered}
\left\|G_{\Phi} N(u)\right\|_{X^{k}} \leqq \alpha\|\Phi\|_{X_{0}^{k}}+\beta\left(T,\|u\|_{X^{1}}\right)\|u\|_{X^{k}} \\
\left\|G_{\Phi} N(u)-G_{\Phi} N\left(u^{\prime}\right)\right\|_{X^{k}} \leqq \beta\left(T,\|u\|_{X^{k}}+\left\|u^{\prime}\right\|_{X^{k}}\right)\left\|u-u^{\prime}\right\|_{X^{k}}
\end{gathered}
$$

for some $\alpha>0$, and $\beta(T, r)=C\left\{T^{1 / 4}+T\right\}\left\{r+r^{2}\right\}$. We choose $R>0$ and $T_{k}=$ $T_{k}(R)>0$ so that

$$
\begin{gathered}
\|\Phi\|_{X_{0}^{k}}<R, \\
\beta\left(T_{k}, 4 \alpha R\right)<1 / 2 .
\end{gathered}
$$

Let $B^{k}\left(T_{k}\right)$ be the closed ball of center 0 and radius $2 \alpha R$ in the space $X^{k}\left(T_{k}\right)$. Then by (3.26) and (3.27), $G_{\Phi} N(\cdot)$ is a contraction mapping of $B^{k}\left(T_{k}\right)$ into itself. Therefore the equation $u=G_{\Phi} N(u)$ has a unique solution $u$ in $B^{k}\left(T_{k}\right)$. Since $T_{k}$ depends only on $k$ and $R$, a standard continuation argument shows that there exists a $T_{\max }>0$ such that $u$ extends to a unique solution in $X^{k}(T)$ for all $T \in\left(0, T_{\max }\right)$, and furthermore that if $T_{\max }<\infty$, then

$$
\varlimsup_{t \uparrow T \text { max }}\|u(t)\|_{X_{0}^{k}}=\infty
$$

We claim that (3.25) holds where the smaller norm in $X_{0}^{1}$ is used. For, otherwise there would be a constant $L>0$ such that

$$
\|u(t)\|_{X_{0}^{1}}<L
$$

for $0 \leqq t<T_{\max }$. By (3.26) and (3.27) with $k=1$, there exists $T_{1}>0$ depending only on $L$ such that the equation $v=G_{v} N(v)$ has a unique solution $v \in B^{1}\left(T_{1}\right)$ for all $\Psi \in X_{0}^{1}$ with $X_{0}^{1}$-norm $<L$. Let $0<\delta<T_{1}$ and $T_{*}=T_{\max }-\delta$. Let $u_{*}$ be the unique solution of $u_{*}=G_{u\left(T_{*}\right)} N\left(u_{*}\right)$ in $B^{1}\left(T_{1}\right)$. Then $u_{*}$ coincides with $u\left(\cdot+T_{*}\right)$ on $[0, \delta)$, so that $u_{*} \in X^{k}(T)$ for all $T$ in $(0, \delta)$. Moreover, (3.26) with $\Phi$ replaced by $u\left(T_{*}\right)$ and $u$ by $u_{*}$ is valid for $T \in(0, \delta)$. It implies that

$$
\begin{aligned}
\left\|u_{*}\right\|_{X^{k}(T)} & =\left\|G_{u\left(T_{*}\right)} N\left(u_{*}\right)\right\|_{X^{k}(T)} \\
& \leqq \alpha\left\|u\left(T_{*}\right)\right\|_{X_{0}^{k}}+\beta\left(T,\left\|u_{*}\right\|_{X^{1}(T)}\right)\left\|u_{*}\right\|_{X^{k}(T)} \\
& \leqq \alpha\left\|u\left(T_{*}\right)\right\|_{X_{0}^{k}}+\beta(\delta, 2 \alpha L)\left\|u_{*}\right\|_{X^{k}(T)} .
\end{aligned}
$$


If we choose $\delta$ so small that $\beta(\delta, 2 \alpha L)<1 / 2$, this inequality yields

$$
\left\|u_{*}\right\|_{X^{k}(T)}<2 \alpha\left\|u\left(T_{*}\right)\right\|_{X_{0}^{k}}
$$

for $0<T<\delta$. But this implies

$$
\varlimsup_{t \uparrow T}\|u(t)\|_{X_{0}^{k}} \leqq 2 \alpha\left\|u\left(T_{*}\right)\right\|_{X_{0}^{k}},
$$

which contradicts (3.30). This proves (3.25).

To prove the last part of the lemma, it is sufficient to show that $u_{n} \rightarrow u$ in $X^{k}\left(T_{k}\right)$, with $T_{k}$ as above. Let $n$ be sufficiently large so that (3.28) with $\Phi$ replaced by $\Phi_{n}$ holds. The above existence argument shows that both $u, u_{n}$ belong to $B^{k}\left(T_{k}\right)$. Then using Lemma 3.2 and (3.20), we have

$$
\begin{aligned}
\left\|u-u_{n}\right\|_{X^{k}\left(T_{k}\right)} & =\left\|G_{\Phi} N(u)-G_{\Phi_{n}} N\left(u_{n}\right)\right\|_{X^{k}\left(T_{k}\right)} \\
& \leqq \alpha\left\|\Phi-\Phi_{n}\right\|_{X_{0}^{k}}+\beta\left(T_{k},\|u\|_{X^{k}}+\left\|u_{n}\right\|_{X^{k}}\right)\left\|u-u_{n}\right\|_{X^{k}\left(T_{k}\right)} \\
& \leqq \alpha\left\|\Phi-\Phi_{n}\right\|_{X_{0}^{k}}+\beta\left(T_{k}, 4 \alpha R\right)\left\|u-u_{n}\right\|_{X^{k}\left(T_{k}\right)}
\end{aligned}
$$

Together with (3.29), this implies that $u_{n} \rightarrow u$ in $X^{k}\left(T_{k}\right)$.

Proof of Lemma 3.1. By Lemma 3.4, there exists a unique solution $(A, \psi)$ of the approximate system (3.3)-(3.5) such that

$$
\left(A, \partial_{t} A, \psi\right) \in C\left([0, T) ; H^{k} \times H^{k-1} \times H^{k}\right), \quad \nabla \psi \in L^{2}\left([0, T) ; H^{k}\right),
$$

for some $T>0$. Moreover, $T=\infty$ if $\left(A, \partial_{t} A, \psi\right)$ does not blow up in the $H^{1} \times$ $L^{2} \times H^{1}$-norm in finite time. Thus to complete the proof, it is sufficient to show that (3.7) holds a priori. In view of the last part of Lemma 3.4, we may assume that $A$ and $\psi$ are smooth.

Let $A_{0}$ be defined by (3.1) and let $Q$ and $E$ be the charge and the energy defined in Sect. 2. Thus $Q(0)=\|\phi\|_{2}^{2}$, and $E(0)$ is estimated as

$$
\begin{aligned}
E(0) & =\|(\nabla-i a) \phi\|_{2}^{2}+\frac{1}{2}\left\|\nabla \Delta^{-1}|\phi|^{2}-b\right\|_{2}^{2}+\frac{1}{4}\|\nabla \times a\|_{2}^{2} \\
& \leqq\left\{\|\nabla \phi\|_{2}+\|a\|_{4}\|\phi\|_{4}\right\}^{2}+C\left\{\|\phi\|_{3}\|\phi\|_{2}+\frac{1}{2}\|b\|_{2}\right\}^{2}+\frac{1}{4}\|\nabla \times a\|_{2}^{2} \\
& \leqq C\left(\|a\|_{1,2},\|b\|_{2},\|\phi\|_{1,2}\right),
\end{aligned}
$$

where we have used the boundedness of $\nabla \Delta^{-1}$ as an operator from $L^{6 / 5}$ to $L^{2}$. Since $A_{\mu}$ and $\psi$ satisfy the regularized system (2.3)-(2.4), Lemma 2.1 implies that $E(t) \leqq E(0)$ and $Q(t) \leqq Q(0)$. Using the Coulomb gauge condition, we have

$$
\begin{aligned}
\frac{1}{2} \frac{d}{d t} \int A_{j} A_{j} d x & =\int \partial_{0} A_{j} A_{j} d x=\int\left\{F_{0 j}+\partial_{j} A_{0}\right\} A_{j} d x \\
& =\int\left\{F_{0 j} A_{j}-A_{0} \partial_{j} A_{j}\right\} d x=\int F_{0 j} A_{j} d x \\
& \leqq 2^{1 / 2} E(0)^{1 / 2}\|A\|_{2} .
\end{aligned}
$$

By integration, this implies

$$
\|A(t)\|_{2} \leqq\|a\|_{2}+2^{1 / 2} E(0)^{1 / 2} t
$$


which proves the first estimate in (3.7). In the Coulomb gauge, the energy for the field A may be rewritten as

$$
\begin{aligned}
\frac{1}{2} \int F_{\mu \nu} F_{\mu \nu} d x & =\int\left\{\partial_{\mu} A_{\nu} \partial_{\mu} A_{v}-\partial_{\mu} A_{v} \partial_{v} A_{\mu}\right\} d x \\
& =\int\left\{\partial_{\mu} A_{v} \partial_{\mu} A_{v}-\partial_{0} A_{0} \partial_{0} A_{0}-2 \partial_{j} A_{0} \partial_{0} A_{j}-\partial_{j} A_{k} \partial_{k} A_{j}\right\} d x \\
& =\int\left\{\partial_{\mu} A_{j} \partial_{\mu} A_{j}+\partial_{j} A_{0} \partial_{j} A_{0}+2 A_{0} \partial_{0} \partial_{j} A_{j}+A_{k} \partial_{k} \partial_{j} A_{j}\right\} d x \\
& =\int\left\{\partial_{\mu} A_{j} \partial_{\mu} A_{j}+\partial_{j} A_{0} \partial_{j} A_{0}\right\} d x
\end{aligned}
$$

It follows that

$$
\sum_{\mu}\left\|\partial_{\mu} A(t)\right\|_{2}^{2}+\sum_{j}\left\|\partial_{j} A_{0}(t)\right\|^{2} \leqq E(0),
$$

so that the second estimate in (3.7) holds. We also have

$$
\begin{aligned}
\left\|\partial_{j} \psi\right\|_{2} & \leqq\left\|D_{j} \psi\right\|_{2}+\left\|A_{j}\right\|_{6}\|\psi\|_{3} \\
& \leqq\left\|D_{j} \psi\right\|_{2}+C \sum_{k=1}^{3}\left\|\partial_{k} A_{j}\right\|_{2} \sum_{k=1}^{3}\left\|\partial_{k} \psi\right\|_{2}^{1 / 2}\|\psi\|_{2}^{1 / 2} \\
& \leqq E(0)^{1 / 2}+C E(0)^{1 / 2} Q(0)^{1 / 4} \sum_{k=1}^{3}\left\|\partial_{k} \psi\right\|_{2}^{1 / 2},
\end{aligned}
$$

where we have used (3.32). Thus

$$
\sum_{j}\left\|\partial_{j} \psi(t)\right\|_{2} \leqq C(Q(0), E(0)),
$$

which together with $\|\psi(t)\|_{2}^{2} \leqq Q(0)$ proves the last estimate in (3.7).

\section{Global MS Field in the Coulomb Gauge}

We now prove the existence of global finite energy solutions of the initial value problem for the exact MS system in the Coulomb gauge, Eqs. (3.3)-(3.4) with $\varepsilon=0$, by making use of Lemma 3.1 and a compactness method.

Theorem 4.1. Assume that $(a, b, \phi) \in H^{1} \times L^{2} \times H^{1}$ and $a, b$ satisfy (3.6). Then there exists at least one solution $(A, \psi)$ of (3.3)-(3.5) with $\varepsilon=0$, such that

$$
A \in C_{w}\left(\mathbb{R}^{+} ; L^{2}\right), \quad \partial_{\mu} A \in B C_{w}\left(\mathbb{R}^{+} ; L^{2}\right), \quad \psi \in B C_{w}\left(\mathbb{R}^{+} ; H^{1}\right) .
$$

Theorem 4.2. Let $A, \psi$ be as in Theorem 4.1. If we define $A_{0}$ by (3.1), then $A_{0}, A, \psi$ satisfy the MS system in its original form (2.1)-(2.2).

Proof of Theorem 4.1. Let $\varepsilon_{n}>0$ with $\varepsilon_{n} \rightarrow 0$. By Lemma 3.1, for each $\mathrm{n}$ there is a solution $\left(\psi_{n}, A_{n}\right)$ of the regularized equations

$$
\begin{gathered}
\square A_{n}=-P J\left(A_{n}, \psi_{n}\right), \quad \nabla \cdot A_{n}=0, \\
\partial_{0} \psi_{n}-\left(i+\varepsilon_{n}\right) \Delta \psi_{n}=K_{\varepsilon_{n}}\left(A_{n}, \psi_{n}\right),
\end{gathered}
$$

such that $\left(A_{n}, \partial_{0} A_{n}, \psi_{n}\right)$ belongs to $C\left(\mathbb{R}^{+}: H^{1} \times L^{2} \times H^{1}\right)$ and assumes the initial value $(a, b, \phi)$ at $t=0$. Moreover, we have

$$
\begin{gathered}
\left\|A_{n}(t)\right\|_{2}<C\{1+t\}, \quad\left\|\partial_{\mu} A_{n}(t)\right\|_{1,2}<C, \\
\left\|\psi_{n}(t)\right\|_{1,2}<C,
\end{gathered}
$$


where in the following discussion $C$ denotes various constants independent of $t$ and $n$. Now from the definition of $J=\left(J_{1}, J_{2}, J_{3}\right)$ in Sect. 2,

$$
\begin{aligned}
\left\|J\left(\psi_{n}, A_{n}\right)\right\|_{3 / 2} & \leqq 2\left\{\left\|\psi_{n}\right\|_{6}\left\|\nabla \psi_{n}\right\|_{2}+\left\|A_{n}\right\|_{6}\left\|\psi_{n}\right\|_{6}^{2}\right\} \\
& \leqq C\left\{1+\left\|\nabla A_{n}\right\|_{2}\right\}\left\|\psi_{n}\right\|_{1,2}^{2} .
\end{aligned}
$$

By (4.4) and (4.5), this implies

$$
\left\|J\left(\psi_{n}, A_{n}\right)(t)\right\|_{3 / 2}<C .
$$

Also, the three estimates above (3.24) give

$$
\left\|K_{\varepsilon_{n}}\left(\psi_{n}, A_{n}\right)\right\|_{3 / 2} \leqq C\left\{\left\|\psi_{n}\right\|_{1,2}^{2}+\left\|\nabla A_{n}\right\|_{2}+\left\|\nabla A_{n}\right\|_{2}^{2}\right\}\left\|\psi_{n}\right\|_{1,2},
$$

which shows that

$$
\left\|K_{\varepsilon_{n}}\left(\psi_{n}, A_{n}\right)(t)\right\|_{3 / 2}<C .
$$

By (4.4)-(4.7), we can extract a subsequence of $\left\{\left(A_{n}, \psi_{n}\right)\right\}$, which we denote again by $\left\{\left(A_{n}, \psi_{n}\right)\right\}$, such that

$$
\begin{gathered}
A_{n} \rightarrow A \text { weakly* in } L^{\infty}\left([0, T] ; H^{1}\right), \quad 0<T<\infty, \\
\partial_{0} A_{n} \rightarrow \partial_{0} A \text { weakly }{ }^{*} \text { in } L^{\infty}\left(\mathbb{R}^{+} ; L^{2}\right), \\
\psi_{n} \rightarrow \psi \text { weakly* in } L^{\infty}\left(\mathbb{R}^{+} ; H^{1}\right), \\
J\left(\psi_{n}, A_{n}\right) \rightarrow \beta \text { weakly* in } L^{\infty}\left(\mathbb{R}^{+} ; L^{3 / 2}\right), \\
K_{\varepsilon_{n}}\left(\psi_{n}, A_{n}\right) \rightarrow \alpha \text { weakly }{ }^{*} \text { in } L^{\infty}\left(\mathbb{R}^{+} ; L^{3 / 2}\right),
\end{gathered}
$$

for some $A, \psi$ satisfying (4.1) and $\alpha, \beta \in L^{\infty}\left(\mathbb{R}^{+} ; L^{3 / 2}\right)$. Passing to the limit $n \rightarrow \infty$ in (4.2) and (4.3), and using (4.8) and (4.10)-(4.12), we have

in $\mathscr{D}^{\prime}\left(\mathbb{R}^{+} ; H^{-1}\right)$.

$$
\begin{gathered}
\square A=-P \beta, \quad \nabla \cdot A=0, \\
\partial_{0} \psi-i \Delta \psi=\alpha,
\end{gathered}
$$

We shall show that $(\psi, A)$ is the desired solution. Now $\partial_{0} A$ is bounded from $\mathbb{R}^{+}$to $L^{2}$, and $\partial_{0}^{2} A, \partial_{0} \psi$ are bounded from $\mathbb{R}^{+}$to $H^{-1}$ by $(4.13)$ and $(4.14)$. Thus $\left(A, \partial_{0} A, \psi\right)$ is continuous as a mapping of $\mathbb{R}^{+}$to $L^{2} \times H^{-1} \times H^{-1}$. Also it is locally bounded from $\mathbb{R}^{+}$to $H^{1} \times L^{2} \times H^{1}$. Thus it follows that $\left(A, \partial_{0} A, \psi\right)$ is weakly continuous as a mapping of $\mathbb{R}^{+}$to $H^{1} \times L^{2} \times H^{1}$.

We claim that the field $(A, \psi)$ satisfies the initial condition (3.5). In fact, if $\langle\cdot, \cdot\rangle$ denotes the duality pairing, $A_{n}$ satisfies

and

$$
\int_{0}^{t}\left\langle A_{n}(s) \partial_{0} f(s)+\partial_{0} A_{n}(s) f(s), v\right\rangle d s=\langle a, v\rangle,
$$

$$
\int_{0}^{t}\left\langle\partial_{t} A_{n}(s) \partial_{0} f(s)+\left\{\Delta A_{n}(s)+P J\left(A_{n}, \psi_{n}\right)(s)\right\} f(s), w\right\rangle d s=\langle b, w\rangle,
$$

for all $(v, w) \in L^{2} \times H^{1}$ and all $f \in C^{\infty}\left(\mathbb{R}^{+}\right)$with $f(0)=1$ and $f(t)=0$ for $t$ large. Taking the limit $n \rightarrow \infty$ and using (4.8), (4.9), (4.11) and (4.13), we find that

$$
\int_{0}^{t}\left\{A(s) \partial_{0} h(s)+\partial_{0} A(s) h(s)\right\} d s=a
$$




$$
\int_{0}^{t}\left\{\partial_{0} A(s) \partial_{0} h(s)+\partial_{0}^{2} A(s) h(s)\right\} d s=b,
$$

as integrals in $L^{2}$ and $H^{-1}$, respectively. This implies $\left.\left(A, \partial_{0} A\right)\right|_{t=0}=(a, b)$. Applying the same argument to $\psi_{n}$, we deduce that $\left.\psi\right|_{t=0}=\phi$.

We also have $\alpha=K_{0}(A, \psi)$ and $\beta=J(A, \psi)$. To see this, let $I$ be a bounded interval in $\mathbb{R}^{+}$, and $\Omega$ a bounded open set in $\mathbb{R}^{3}$. It is sufficient to show that $\alpha$ and $\beta$ coincide with $K_{0}(A, \psi)$ and $J(A, \psi)$ on $I \times \Omega$, respectively. Now by (4.4), $\left\{\left(A_{n}, \partial_{0} A_{n}\right)\right\}$ is a bounded sequence in $L^{4}\left(I ; H^{1}(\Omega) \times L^{2}(\Omega)\right)$. Since $H^{1}(\Omega) \subset L^{4}(\Omega) \subset L^{2}(\Omega)$, with the first imbedding compact and the second one continuous, a standard compactness lemma [2] then asserts that there is a subsequence of $\left\{A_{n}\right\}$, again denoted by $\left\{A_{n}\right\}$, such that

$$
A_{n} \rightarrow A \text { strongly in } L^{4}(I \times \Omega) \text {. }
$$

Similarly, since $\left\{\partial_{0} \psi_{n}\right\}$ is bounded in $L^{\infty}\left(\mathbb{R}^{+} ; H^{-1}\right)$ as indicated above, and so $\left\{\left(\psi_{n}, \partial_{0} \psi_{n}\right)\right\}$ is a bounded sequence in $L^{2}\left(I ; H^{1}(\Omega) \times H^{-1}(\Omega)\right)$, we may assert that

$$
\psi_{n} \rightarrow \psi \text { strongly in } L^{4}(I \times \Omega) .
$$

By (4.5), $\left|\psi_{n}\right|^{2}$ is bounded in $L^{2}\left(I ; L^{6 / 5}\right)$, while by (4.16) and the arbitrariness of $\Omega$, we may assume $\psi_{n} \rightarrow \psi$ a.e. on $I \times \mathbb{R}^{3}$. It follows that $\left|\psi_{n}\right|^{2} \rightarrow|\psi|^{2}$ weakly in $L^{2}\left(I ; L^{6 / 5}\right)$, and this implies

$$
\Delta^{-1}\left|\psi_{n}\right|^{2} \rightarrow \Delta^{-1}|\psi|^{2} \text { weakly in } L^{2}\left(I ; L^{6}\right),
$$

since $\Delta^{-1}$ is bounded from $L^{6 / 5}$ to $L^{6}$. Therefore by (4.8), (4.10) and (4.15)-(4.17), we have

$$
\begin{aligned}
J\left(A_{n}, \psi_{n}\right) & \rightarrow J(A, \psi) \text { weakly in } L^{4 / 3}(I \times \Omega), \\
K_{\varepsilon_{n}}\left(A_{n}, \psi_{n}\right) & \rightarrow K_{0}(A, \psi) \text { weakly in } L^{4 / 3}(I \times \Omega) .
\end{aligned}
$$

Thus $\alpha=K_{0}(A, \psi)$ and $\beta=J(A, \psi)$ on $I \times \Omega$, which completes the proof.

Proof of Theorem 4.2. Let $A_{0}$ be defined by (3.1), that is, $\triangle A_{0}=J_{0}$. Then $A_{0}, A, \psi$ clearly satisfy the component of (2.1) with $\mu=0$ and also (2.2). To see that (2.1) also holds for $\mu \neq 0$, we use the following fact. If $f \in H^{1}$ and $g \in H^{-1}$, and if $s>3 / 2$, then the inequality

$$
|\langle g, \zeta f\rangle| \leqq C\|g\|_{-1,2}\|f\|_{1,2}\|\zeta\|_{s, 2}
$$

holds for all $\zeta \in H^{s}$. Hence the product $f g$ is well-defined as an element of $H^{-s}$. Since $\psi$ is in $L^{\infty}\left(\mathbb{R}^{+} ; H^{1}\right)$ and $D_{0} \psi=i D_{j} D_{j} \psi$ is in $L^{\infty}\left(\mathbb{R}^{+} ; H^{-1}\right)$, it follows that

$$
\partial_{0} J_{0}=2 \operatorname{Re}\left\{\bar{\psi} D_{0} \psi\right\}=2 \operatorname{Re}\left\{i \bar{\psi} D_{j} D_{j} \psi\right\}=\partial_{j} J_{j},
$$

by the definition of $J_{\mu}$ in Sect. 2. Since $A_{0}=\Delta^{-1} J_{0}$, this implies

$$
\partial_{0} A_{0}=\partial_{k} \Delta^{-1} J_{k}
$$

From $(3.3),(4.18)$ and the definition of $P$, it follows that

$$
\partial^{\mu} F_{\mu j}=\square A_{j}-\partial_{j} \partial_{0} A_{0}=-(P J)_{j}-\partial_{j} \partial_{k} \Delta^{-1} J_{k}=J_{j},
$$

as was to be shown. 


\section{Global Solutions in Other Gauges}

In this section we consider the initial value problem for the MS system (2.1)-(2.2) in the Lorentz gauge $\partial^{\mu} A_{\mu}=0$ and in the temporal gauge $A_{0}=0$.

The problem in the Lorentz gauge reduces to the system

$$
\begin{gathered}
\square A_{v}+J_{v}=0, \quad \partial^{\mu} A_{\mu}=0, \\
i D_{0} \psi+D_{j} D_{j} \psi=0, \\
\left.\left(A_{\mu}, \partial_{0} A_{\mu}, \psi\right)\right|_{t=0}=\left(a_{\mu}, b_{\mu}, \phi\right) .
\end{gathered}
$$

The equations require us to assume the initial constraints

$$
b_{0}=\partial_{j} a_{j}, \quad \Delta a_{0}=\partial_{j} b_{j}+|\phi|^{2} .
$$

Theorem 5.1. (Lorentz gauge) Assume that $\left(a_{\mu}, b_{\mu}, \phi\right) \in H^{1} \times L^{2} \times H^{1}$ and the $a_{\mu}, b_{\mu}$ satisfy (5.4). Then the initial value problem (5.1)-(5.3) has a solution $\left(\psi, A_{\mu}\right)$ such that

$$
\left(A_{0}, \partial_{0} A_{0}\right) \in C\left(\mathbb{R}^{+} ; H^{1} \times L^{2}\right), \quad\left(A_{j}, \partial_{0} A_{j}, \psi\right) \in C_{w}\left(\mathbb{R}^{+} ; H^{1} \times L^{2} \times H^{1}\right) .
$$

Proof. We will reduce the problem to the Coulomb gauge by a gauge transformation. Let $a, b$ denote the 3 -vectors with components $a_{j}, b_{j}$, respectively. Let $f=\Delta^{-1} \nabla \cdot a$. We define

$$
\left(a^{c}, b^{c}, \phi^{c}\right)=\left(P a, P b, e^{-i f} \phi\right),
$$

where $P v=v-\nabla\left(\nabla \cdot \Delta^{-1} v\right)$ as before. Note that $\phi^{c} \in H^{1}$, since $\nabla f=(I-P) a$. By Theorem 4.1, the MS system in the Coulomb gauge, (3.3)-(3.4) with $\varepsilon=0$, has a solution $\left(A^{c}, \psi^{c}\right), A^{c}=\left(A_{1}^{c}, A_{2}^{c}, A_{3}^{c}\right)$, such that

$$
A^{c} \in C_{w}\left(\mathbb{R}^{+} ; L^{2}\right), \quad \partial_{\mu} A^{c} \in B C_{w}\left(\mathbb{R}^{+} ; L^{2}\right), \quad \psi^{c} \in B C_{w}\left(\mathbb{R}^{+} ; H^{1}\right),
$$

that satisfies the initial condition

$$
\left.\left(\psi^{c}, \partial_{0} A^{c}, A^{c}\right)\right|_{t=0}=\left(a^{c}, b^{c}, \phi^{c}\right) .
$$

Let

$$
A_{0}^{c}=\Delta^{-1}\left|\psi^{c}\right|^{2}
$$

We define $\lambda$ to be the solution of the initial value problem

$$
\square \lambda=-\partial_{0} A_{0}^{c},\left.\quad\left(\lambda, \partial_{0} \lambda\right)\right|_{t=0}=(f, g),
$$

where $f$ is as above and $g=\Delta^{-1} \nabla \cdot b$, so that $\nabla f \in H^{1}$ and $\nabla g \in L^{2}$. By (4.18),

$$
\partial_{0} A_{0}^{c}=\Delta^{-1} \nabla \cdot J^{c},
$$

where $J^{c}=J\left(A^{c}, \psi^{c}\right) \in L^{\infty}\left(\mathbb{R}^{+} ; L^{6 / 5}\right)$. Since $\Delta^{-1} \nabla$ is bounded from $L^{6 / 5}$ to $L^{2}$, this implies that $\partial_{0} A_{0}^{c}$ is in $L^{\infty}\left(\mathbb{R}^{+} ; L^{2}\right)$. Then the energy estimate for (5.7) shows that $\nabla \lambda \in C\left(\mathbb{R}^{+} ; L^{2}\right)$ and $\partial_{0} \lambda \in C\left(\mathbb{R}^{+} ; L_{1 o c}^{2}\right)$. We define $A_{\mu}$ and $\psi$ by the gauge transformation

$$
A_{\mu}=A_{\mu}^{c}+\partial_{\mu} \lambda, \quad \psi=e^{i \lambda} \psi^{c} .
$$


We shall show that $\left(A_{\mu}, \psi\right)$ is the desired solution of (5.1)-(5.3). Now from (5.6)-(5.8) and (5.4), we have

$$
\begin{aligned}
\left.A_{0}\right|_{t=0} & =\left.A_{0}^{c}\right|_{t=0}+g=\Delta^{-1}\left|\phi^{c}\right|^{2}+\nabla \cdot \Delta^{-1} b=a_{0}, \\
\left.\partial_{0} A_{0}\right|_{t=0} & =\left.\partial_{0} A_{0}^{c}\right|_{t=0}+\left.\partial_{0}^{2} \lambda\right|_{t=0}=\left.\Delta \lambda\right|_{t=0}=\nabla \cdot a=b . \\
\left.A_{j}\right|_{t=0} & =a_{j}^{c}+\partial_{j} f=(P a)_{j}+((I-P) a)_{j}=a_{j}, \\
\left.\partial_{0} A_{j}\right|_{t=0} & =b_{j}^{c}+\partial_{j} g=(P b)_{j}+((I-P) b)_{j}=b_{j}, \\
\left.\psi\right|_{t=0} & =e^{i f} \psi^{c}=\phi .
\end{aligned}
$$

Thus $\left(A_{\mu}, \psi\right)$ satisfies (5.3). Applying $\partial^{\mu}$ to the first equation in (5.8), and using (5.7) and $\partial_{j} A_{j}^{c}=0$, we see that the $A_{\mu}$ satisfy the Lorentz gauge condition $\partial^{\mu} A_{\mu}=0$.

We now prove (5.5) and (5.1)-(5.3). From the definition (5.8), $A_{\mu}$ and $\psi$ belong to $C_{w}\left(\mathbb{R}^{+} ; L^{2}\right)$. From $(5.6)-(5.8)$, we have

$$
\square A_{0}=-\left|\psi^{c}\right|^{2} .
$$

Since $\left.\left(A_{0}, \partial_{0} A_{0}\right)\right|_{t=0}=\left(a_{0}, b_{0}\right) \in H^{1} \times L^{2}$, this equation implies that $A_{0} \in$ $C\left(\mathbb{R}^{+} ; H^{1}\right)$ with $\partial_{0} A_{0} \in C\left(\mathbb{R}^{+} ; L^{2}\right)$. Then $\partial_{j} A_{j} \in C\left(\mathbb{R}^{+} ; L^{2}\right)$, since $\partial^{\mu} A_{\mu}=0$. We also have

$$
\partial_{j} A_{k}-\partial_{k} A_{j}=\partial_{j} A_{k}^{c}-\partial_{k} A_{j}^{c} \in C_{w}\left(\mathbb{R}^{+} ; L^{2}\right) .
$$

The last two facts imply that $\partial_{k} A_{j} \in C_{w}\left(\mathbb{R}^{+} ; L^{2}\right)$ for all $j$ and $k$. Similarly,

$$
\partial_{0} A_{j}=\partial_{0} A_{j}^{c}-\partial_{j} A_{0}^{c}+\partial_{j} A_{0} \in C_{w}\left(\mathbb{R}^{+} ; L^{2}\right) .
$$

Thus we have proved that $A_{\mu}, \partial_{v} A_{\mu} \in C_{w}\left(\mathbb{R}^{+} ; L^{2}\right)$. In particular, $A_{j} \in C_{w}\left(\mathbb{R}^{+} ; H^{1}\right)$. By (5.8), this implies $\partial_{j} \lambda \in C_{w}\left(\mathbb{R}^{+} ; H^{1}\right)$. Then

$$
\partial_{j} \psi=e^{i \lambda}\left\{\partial_{j}+i \partial_{j} \lambda\right\} \psi^{c} \in L_{1 o c}^{\infty}\left(\mathbb{R}^{+} ; L^{2}\right) .
$$

Thus $\psi \in L_{\text {loc }}^{\infty}\left(\mathbb{R}^{+} ; H^{1}\right)$. Now by Theorem 4.2, $\left(A_{\mu}^{c}, \psi^{c}\right)$ satisfies (2.1) and (2.2), which are invariant under the gauge transformation (5.8). Thus $\left(A_{\mu}, \psi\right)$ also satisfies (2.1)-(2.2) and hence (5.1)-(5.2) since $\partial^{\mu} A_{\mu}=0$. The weak continuity of $\psi$ in $t$ now follows from (5.2). This completes the proof.

In the temporal gauge $A_{0}=0$, we may write the initial value problem for the MS system as

$$
\begin{gathered}
-\partial_{0} \partial_{j} A_{j}=J_{0}, \quad A_{0}=0, \\
\square A_{j}+\partial_{j} \partial_{k} A_{k}=-J_{j}, \\
i \partial_{0} \psi+D_{j} D_{j} \psi=0, \\
\left.\left(A_{j}, \partial_{0} A_{j}, \psi\right)\right|_{t=0}=\left(a_{j}, b_{j}, \phi\right) .
\end{gathered}
$$

The initial constraint is

$$
\partial_{j} b_{j}+|\phi|^{2}=0 .
$$

Theorem 5.2. (Temporal gauge) Assume that $\left(a_{j}, b_{j}, \phi\right) \in H^{1} \times L^{2} \times H^{1}$ and the $b_{j}$ satisfy (5.13). Then the initial value problem (5.9)-(5.13) has a solution $(A, \psi)$ such that

$$
A_{j} \in C_{w}\left(\mathbb{R}^{+} ; H^{1}\right), \quad \partial_{0} A_{j} \in B C_{w}\left(\mathbb{R}^{+} ; L^{2}\right), \quad \psi \in B C_{w}\left(\mathbb{R}^{+} ; H^{1}\right) .
$$


Proof. We define $f, a^{c}, b^{c}, \phi^{c}, A_{0}^{c}, A^{c}$ and $\psi^{c}$ as in the proof of Theorem 5.1. Using the solution $\lambda$ of the simple equation

$$
\partial_{0} \lambda+A_{0}^{c}=0,\left.\quad \lambda\right|_{t=0}=f,
$$

we define $\psi, A_{\mu}$ by $(5.8)$. Then $\left(\psi, A_{j}\right)$ provides the solution of the theorem.

\section{References}

1. Healy, W.: Non-relativistic quantum electrodynamics. New York: Academic Press, 1982

2. Lions, J.-L.: Quelques méthods de résolution des problèmes aux limites non linéaires. Paris: Dunod-Gauthier Villars, 1969

3. Nakamitsu, K., Tsutsumi, M.: The Cauchy problem for the coupled Maxwell-Schrödinger equations. J. Math. Phys. 27, 211-216 (1986)

4. Schiff, L.I.: Quantum mechanics. 2nd ed. New York: McGraw-Hill, 1955

5. Tsutsumi, M., Nakamitsu K.: Global existence of solutions to the Cauchy problem for coupled Maxwell-Schrödinger equations in two space dimensions. In: Lightbourne, J.H., Rankin, S.M. (eds.) Physical mathematics and nonlinear partial differential equations, New York: Marcel Dekker, 1985, pp 139-155

6. Tsutsumi, Y.: Global existence and asymptotic behavior of solutions for the MaxwellSchrödinger equations in three space dimensions. Commun. Math. Phys. 151, 543-576 (1993)

Communicated by H. Araki 